\title{
Low-Voltage Scanning Electron Microscopy of Bovine Gametes - a Review of Preparative Techniques and Methods
}

\author{
M. W. Tengowski* \\ *Global Clinical Technologies, Pfizer Global Research \& Development, 2800 Plymouth Road, \\ MS16-1A/6, Ann Arbor, MI 48105 mark.w.tengowski@pfizer.com
}

Transmission and scanning electron microscopes have been instrumental in studying the fertilization event. However, EM specimen preparation captures static events, a limitation not seen with today's live-cell imaging techniques. The impact of preparative techniques should be considered.

Results into preparative techniques to study fertilization via LVSEM are presented. Control fixation utilized $1 \%$ glutaraldehyde with $1 \%$ formaldehyde in $0.15 \mathrm{M}$ cacodylate buffer (CB, $\mathrm{pH} 7.4)$ overnight at room temperature (RT) followed by a CB wash for $1 \mathrm{~h}$ at RT. A second oocyte group was fixed in $1 \%$ glutaraldehyde with $1 \%$ formaldehyde in $0.15 \mathrm{M} \mathrm{CB}$ overnight (RT) followed by a $1 \mathrm{~h} \mathrm{RT} \mathrm{CB}$ wash and treated with 1\% thiocarbohydrazide for $20 \mathrm{~min}$ before post-fixing in 1\% osmium tetroxide in distilled water for $60 \mathrm{~min}$ at RT. A third oocyte group utilized a conductive staining protocol[1]. These oocytes were pretreated with $0.02 \%$ saponin plus $1 \%$ ruthenium red in $0.15 \mathrm{M} \mathrm{CB}$ for $45 \mathrm{~min}$ at RT before fixation with 1\% glutaraldehyde, $1 \%$ formaldehyde, $0.02 \%$ saponin, and $1 \%$ ruthenium red in $0.15 \mathrm{M} \mathrm{CB}$ overnight followed by a $1 \mathrm{~h} \mathrm{RT} \mathrm{CB}$ wash. These oocytes were washed in $0.02 \%$ saponin plus $1 \%$ ruthenium red in $0.15 \mathrm{M} \mathrm{CB}$ for 60 min at RT prior to post-fixation with $1 \%$ osmium tetroxide, $0.02 \%$ saponin, and $0.75 \%$ ruthenium red in $0.15 \mathrm{M} \mathrm{CB}$ for $2 \mathrm{~h}$ prior to conductive staining with two incubation cycles utilizing $1 \%$ thiocarbohydrazide (T) and $1 \%$ osmium tetroxide $(\mathrm{O})$ in distilled water $(\mathrm{T} / \mathrm{O} / \mathrm{T} / \mathrm{O}$ for 20/60/20/60 min). Samples prepared for LVSEM or cSEM were washed in Tyrode's medium containing no added calcium or protein and allowed to adhere to acetone-cleaned No. 2 coverslips cut to a size of 8 X $20 \mathrm{~mm}$ exposed to 1 $\mathrm{mg} / \mathrm{ml}$ poly-L-lysine[2]. Oocytes were dehydrated with an increasing ethanol concentration prior to critical-point drying[3]. A fourth fixation protocol utilized a high-pressure cryoimmobilization technique followed by freeze-substitution[4] and critical-point dried. Samples were stored in a desiccator until viewing. Platinum was argon ion-beam sputter coated onto samples approximately 1-2 $\mathrm{nm}$ in thickness. This provides adequate coating for the sample when viewed in the Hitachi S900 field-emission LVSEM. This instrument was operated at an accelerating voltage of $1.5 \mathrm{keV}$. The control aldehyde-fixed samples processed for viewing in a Hitachi S-570 (cSEM) were sputtercoated with $30 \mathrm{~nm}$ gold and viewed at either $10 \mathrm{keV}$ or $30 \mathrm{keV}$ accelerating voltage. LVSEM images were captured using Gatan's Digital Micrograph 2.5 and archived on optical disk; cSEM images were recorded on Type 55 Instant Polaroid film.

References

[1] G. Familiari et al., Microscopy Research \& Technique 23 (3) (1992) 225.

[2] D. Mazia et al., Journal of Cell Biology 66 (1) (1975) 198.

[3] H. Ris, Journal of Cell Biology 100 (5) (1985) 1474.

[4] P. Walther et al., Scanning Microscopy 7 (4) (1993) 1283.

[5] M.W. Tengowski and G. Schatten, Microscopy \& Microanalysis 3 (3) (1997) 193.

[6] M.W. Tengowski, Methods in Molecular Biology 253(2004) 165. 


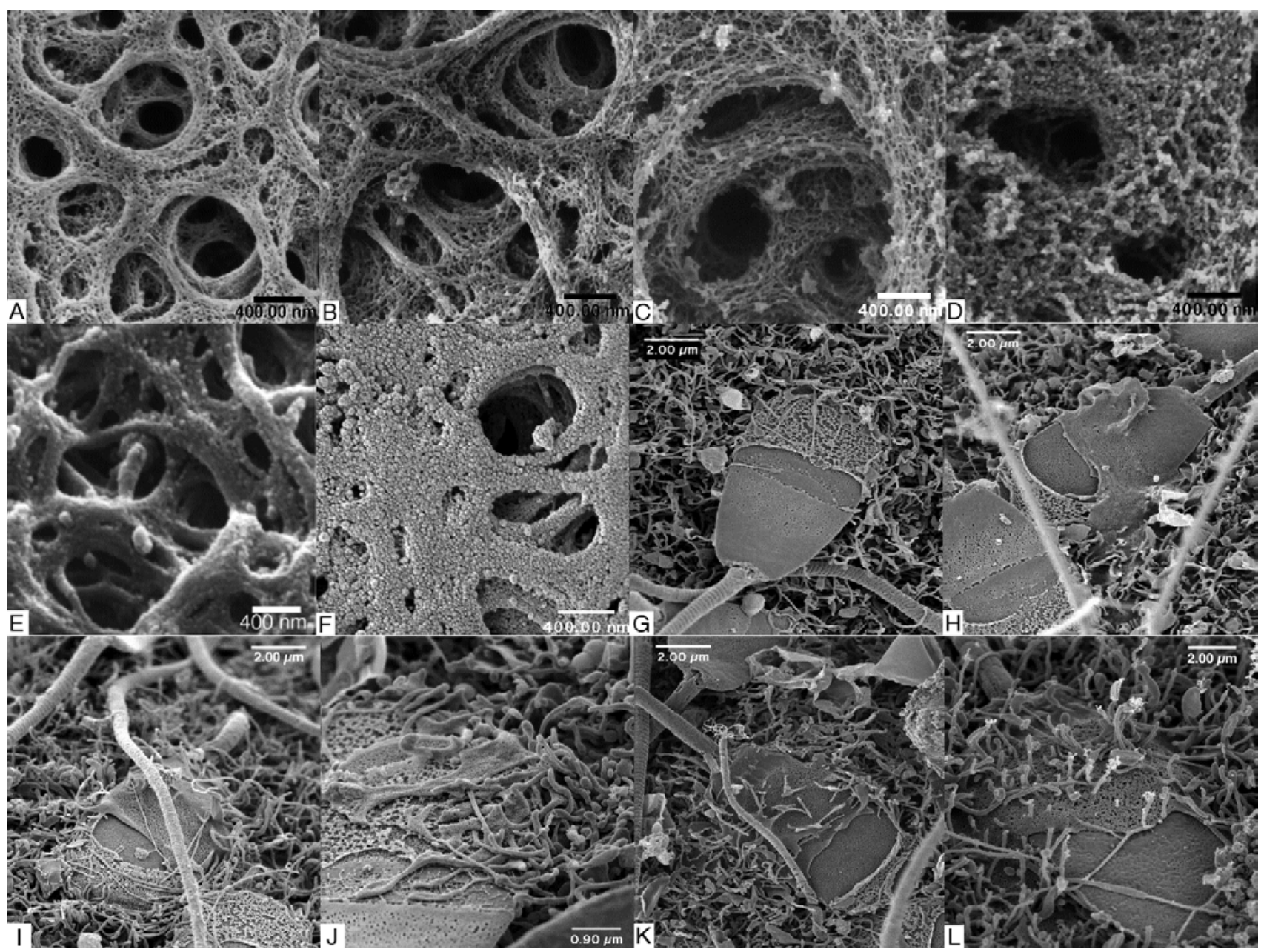

LVSEM of bovine gametes: zona pellucida, sperm incorporation into zona-free oocyte preps

Figure 1. A) Bovine oocyte chemically fixed with glutaraldehyde and formaldehyde without postfixation, B) post-fixed with thiocarbohydrazide/osmium tetroxide, C) cryopreserved by highpressure freezing and freeze-substitution, A-C samples prepared for LVSEM with platinum coating. D) Prepared with the TOTO conductive staining protocol and viewed uncoated in the LVSEM. E) Prepared for cSEM with gold coating and viewed at $30 \mathrm{keV}$ accelerating voltage. F) Sample in E viewed with the LVSEM. G-L) Acrosome-reacted bull sperm on the surface of zona pellucida-free bovine oocytes. G) 10-minute sperm exposure, oocyte microvilli make contact with the sperm over the anterior head in the vesiculated outer acrosomal membrane and equatorial segment membrane areas. Spherical structures apparent around sperm head, most likely cortical granule products. $\mathrm{H}$ ) Microvilli on oolemma surface in contact with sperm head after 20 minutes. This is the earliest time point that membrane fusion is observed via LVSEM. Fusion event begins primarily over the sperm head equatorial segment. I) 30 minutes post sperm addition, the area over the equatorial segment completely fused with the oolemma membrane, yet the oocyte microvilli are seen interacting with the acrosomal and post equatorial domains. A portion of the sperm membrane is still exposed to the media. J) 60 minutes after sperm addition, microvilli from the oocyte can still be seen interacting with the sperm equatorial segment and head. Processes are extended to the equatorial region in many sperm. K, L) The appearance of microvilli over the equatorial and post-equatorial region of an incorporating bull sperm. The sperm tail and implantation fossa are not incorporated at this time point, and the inner acrosomal membrane can still be discerned. Originally published in [5,6]. 